\title{
Emotion Contagion Model for Crowds
}

\author{
Amyr B.F. Neto ${ }^{1}$, Catherine Pelachaud ${ }^{2}$, Soraia R. Musse ${ }^{1}$ \\ ${ }^{1}$ Department of Computer Science - FACIN - PUCRS - Porto Alegre - RS, Brazil \\ Emails: amyr.forteseacad.pucrs.br, soraia.mussedpucrs.br \\ ${ }^{2}$ CNRS-LTCI, Telecom ParisTech - Paris - Île de France, France \\ Email: catherine.pelachaudetelecom-paristech. fr
}

\begin{abstract}
Latest advances in crowd simulation models that attempt to make agents with more realistic human-like behaviors explore heterogeneity of agent behaviors in order to achieve increased overall simulation realism. In general, human behavioral and psychological studies are used as base of knowledge and researchers try to simulate observed human behavior patterns within virtual agents. In this direction, this paper implements an emotion contagion model, within crowd simulation scenarios, in order to create realistic perception of agent behaviors on crowds.
\end{abstract}

Keywords - Crowd Simulation, Emotion Contagion.

\section{INTRODUCTION}

Since pioneer approaches in crowd simulation systems, more effective mathematical models were developed by scientific community. At the same time, computational power has significantly improved. These advances have allowed creation of even more complex crowd simulation models. At the same time, human behavior is object of study for many years in human sciences, such as sociology and psychology. Sociology researchers, such as Melissa Hurst on her lecture [13] have mapped crowd and mass behavior, how they interact with each other and how the concerns of the crowd are communicated and spread along crowd individuals. Also, psychology researchers have mapped traits of human personality, as in [8] and [7], that rule individual behavior and decision making in many daily situations. Yet, researchers like Hatfield [10] have been making series of experiments in order to address emotion and emotion contagion process in humans. Hatfield defines emotion contagion process as a continuous process of mimicry, emotional feedback and self-perception, which drives the expressed emotion of a person or character into others.Computer science groups have mapped these human studies results to computer system parameters in order to simulate more realistic and complex crowd behaviors. Some recent works have mapped emotion contagion process within crowds, but using very simple emotion contagion models [3]. Others have created more complex emotion contagion models, but have applied only to small groups of agents [1]. Since crowd simulation models deal with hundreds or thousands of agents [19], interaction between agents can lead to very big number of calculations in order to compute emotion influence among each other, which in turn may be prohibitive in terms of simulation time in certain crowd scenarios. The objective here is to contextualize emotion contagion computational model within crowd simulation models. The contribution of this paper is to apply an emotion contagion model in the context of crowd simulation and multiple emotions in agents.

The remainder of this paper is organized as follows: in Section 2, relevant studies in crowd simulation are cited. In Section 3, psychological modeling of emotional contagion phenomena is presented. In Section 4, emotion contagion computational model is presented and changes made to apply such a model in crowd simulation scenarios are discussed. Section 5 presents and discusses some results obtained so far. Finally, some directions pointed by the research for future works are commented in Section 6.

\section{RELATED WORK}

Crowd simulation models started with Reynolds flocks and herds [16] and Helbing social forces [11] models. After that, several different approaches were proposed to model crowds. On the work [4], the author proposes an algorithm where the agents are blind, and can only perceive the surrounding environment through markers. Inspired by the algorithm of space colonization [17], [18], each marker, called by the author as an auxin is either walkable or nonwalkable. In the latter case, they might be obstacle, or in use by another agent. This way, as agents move in the environment, they respect other agents spaces, and a collision free navigation is achieved. They also respect obstacles such as walls, that can be described as nonwalkable area. This work was later improved by Hocevar [12] who added group behavior to the model.

Most recently, approaches have been addressing personality models, such as Durupinar and colleagues in [5], where the authors presented an approach to incorporate OCEAN(Openness, Conscientiousness, Extraversion, Agreeableness and Neuroticity) personality model, as proposed by Goldberg [8], into HiDAC (HighDensity Autonomous Crowds) simulation model published by Pelechano, Allbeck and Badler [15]. The objective of this work is to easily create different personalities. To do so, the parameters of the HiDAC simulation model were mapped into individual OCEAN traits and their polarities. Also, simple adjectives such as "leadership", "impatience", "panic" among others are mapped to the same traits. This way, agents behaviors are easily specified through adjectives, which in turn are translated to OCEAN traits, and then to lower level HiDAC parameters. 
Another personality model originated from psychology studies and used in crowd simulation models is Eysenck PEN [7]. An example of work using this model is [9], where the authors conduct two user studies: one to correlate simulation parameters with personality model parameters, and the other to validate the correlations found. By the end, the authors accomplish a high level adjective choice, based on PEN personality trait adjectives, and are able to remap those into lower level agent parameters.

But personalities are very different from emotions. Personalities maps constant personality traits, like Extraversion or Openness, which do not vary in time. On the other hand, emotions have a short time span, and are impacted by agents personality. Emotions were recently applied in crowds by [3], who present a simple emotion model with emotion contagion feature. Despite the ability to simulate emotion contagion, this work focuses on the perception of body expression animation instead. The agents can be either in sad, happy, or neutral emotional status, and they can be either walking or standing. An animation corres-ponding to body expression of such emotions is played according to agents state. The emotion contagion model itself is very simple if compared to other emotion contagion models like [1] and [2], allowing agent to change their status once contagion occurs.

The model presented here can work with only three predetermined emotions namely Sadness, Anger and Joy. The model developed by Bosse and colleagues is adopted in this work. Further detailed explanation about this model can be found in Section 4.

As stated before, unlike personality-based models, emotions are not constant. They depend on agent personality, mood, neighboring agents, surrounding environment and environ-mental events. This way, emotions can vary in time and evolve during the simulation. Emotions, moods, and emotion contagion can be an useful tool to model common, yet abnormal, crowd behavior ssuch a spanic, and conflicting, which can be very useful for predicting behavior abnormalities and preparing contingency plans forthose occurrences. Also, those same abnormal behaviors may result in more realistic agent behavior perception during a computer game or movie animation. This work proposes applying more dynamic and complete emotion contagion model to agents in crowds.

\section{EMOTION CONTAGION PROCESS}

This section depicts emotion contagion process as psychology researchers understands it. According to [10], one's emotional contagion may be experienced by a continuous process of mimicry, emotional feedback and self-perception, which drives the emotion in the affected subject. Those three processes are visceral, automatic, unconscious psychological mechanisms, and are defined as follows.

\section{A. Mimicry}

In conversation people tend automatically and continuously to mimic and synchronize their movements with behaviors of others. This process is mainly induced by facial expression interpretation, but is also influenced by voice pitch and posture, movements, and instrumental behaviors. This synchrony is automatic and almost instantaneous.

\section{B. Emotional Feedback}

Addresses the fact that, as proven by subject studies, one tends to have visceral feedback on expressed emotions. References on studies designed to prove this hypothesis can be found in the work of Hatfield [10]. One of these studies, as an example, was designed to test human subjects while watching a comedy movie. Some subjects were forced to hold a pencil with their teeth in a way the mouth is kept open like a smile, this can be called the smiley group. Other group was asked to hold the pencil with their lips, like a straw, making the subject almost unable to smile, thus notsmiley group. The third group was given a pencil, but no instructions were given regarding the pencil so, mostly, they just held the pencil in their hands, and this is called the neu-tral or control group. The subjects were then asked to answer a questionary to evaluate how funny they perceived the movie. The results of this study show significant higher fun perception in the smiley group scoring much higher (funnier) than the not-smiley group. And yet a difference between the smiley group with significant higher score than the control group, which in turn scored higher than the notsmiley group. This and other studies prove evidence that forcing a facial expression of certain emotion may actually drive such emotion.

\section{Self-perception}

Is the process of perceiving one's own self. This means that individuals draw inferences about their own emotional states based on the emotional expressions and behaviors evoked in them by the emotional states of others. In other words, by perceiving their own facial expression and posture, individuals tend to catch those emotions moment to moment.

Beyond the individual inherent susceptibility, external factors such as interpersonal relationship, and the level in which the emotion is expressed by others influence emotion contagion process. Given the definitions above, the emotion contagion process is defined by i) one subject, either a person or virtual agent, expressing one particular emotion, on a given level of expressiveness, to another subject or group of subjects, ii) the group of subjects exposed to the emotion, each member perceives the emotion at its own perception level, and start mimicry and synchrony process with the perceived emotion, and iii) selfawareness on the influenced subjects drives visceral emotion into them. This process happens continuously each moment of the interaction.

\section{EMOTION CONTAGion COMPUtATIONAL MOdEL}

This section depicts the work of Bosse et. al [2], where the authors proposes a computational model for emotion contagion process. The model does not consider agents mood or emotional tendency as in an emotion model. Instead, it only models overall group emotion influence in 
group agents. In other words, it models only the emotional contagion process, varying agents emotions according to other agents influence. The model considers agents expressiveness, susceptibility to catch others emotion, inter-agent relationship, emotional tendency to absorb or amplify emotions, and group emotional level. It is important to notice that, in the amplification model, an agent can amplify an emotion upward or downward, depending on its bias, this also reflects group average emotion. This enables the model to simulate observed emotion contagion spirals, where emotions tend to increase, or decrease, in intensity due to dyadic interaction. One limitation of the model addressed herein is that the model threats only one non-specified emotion. This could be any emotion, like in [6], which distinguishes anger, disgust, fear, joy, sadness and surprise. Results shows that upward and downward spiral can be observed in simulations, as well as agents influences over each other.

Mathematically, Bosse's model defines the emotion of an agent as a value $q$ in the range $[0,1]$, and represents the intensity of an unspecified emotion. The only restriction about emotion specification is that it should be the same emotion for all agents in the simulation. Each agent expresses its own emotion to the group at a given expression level $\varepsilon$ also in the range $[0,1]$. This way extroverted, expressive, active persons will induce a stronger contagion of an emotion than a shy agent. On the perceiving emotion agent side the authors propose to represent the susceptibility of catching the emotions of others by the variable $\delta$, which also lies in the range $[0,1]$, and defines how much a person allows other people emotions to affect his own emotional state. Finally, the relationship between the agents is taken into consideration. Depending on people relational links, emotion contagion may be stronger or weaker. This interpersonal relationship link is represented in Bosse's model by a variable $\alpha_{\mathrm{kl}}$, which represents the relationship between agents $k$ and $l$. Notice that $\alpha_{k 1}$ can be different from $\alpha_{1 k}$, for example, as the influence of the mother over the son may be different from the influence of the son over the mother during a particular emotion contagion process. This leads to a square matrix containing all $\alpha_{\mathrm{kl}}$ parameters, with $\mathrm{k}$ and 1 in the range [1, $G]$ where $G$ is the number of agents in the group. Also, the main diagonal of this matrix is irrelevant, since it would represent the relationship of an agent with itself, which just makes no sense in this context.

In order to address emotional spirals, two variables were proposed by the authors: i) a bias represented by the variable $\eta$ to define the agents tendency to absorb, which means that the group members converge to some average emotional level, or amplify emotions, which means the group members catch others emotion in a way they generate higher or lower overall emotional energy level, and ii) a bias represented by the variable $\beta$ to decide whether the amplification model tendency is upwards or downwards. Considering a group of two agents with $\mathrm{S}$ being the sender of a particular emotion, $\mathrm{R}$ being the receiver of such emotion, and $j$ being any agent in the group, all those definitions can be summarized in Table 1.
TABLE 1 -VARIABLES TO BE CONSIDERED ON THE EMOTIONAL CONTAGION PROCESS.

\begin{tabular}{|l|l|}
\hline Variable & Purpose \\
\hline$q_{j}$ & Represents the current emotion level of agent $j$. \\
\hline$\varepsilon_{S}$ & Represents agents S expressiveness. \\
\hline$\delta_{R}$ & Represents agents R emotional susceptibility. \\
\hline$\alpha_{S R}$ & $\begin{array}{l}\text { Represents the influence of S over R. Notice that } \alpha_{S R} \\
\text { can be different from } \alpha_{R S} .\end{array}$ \\
\hline$\eta_{j}$ & Bias agent $j$ tendency to amplify or absorb emotions. \\
\hline$\beta_{j}$ & $\begin{array}{l}\text { Given a bias to amplify emotions, this variable bias } \\
\text { agent } j \text { tendency to amplify emotions upward or } \\
\text { downward. }\end{array}$ \\
\hline
\end{tabular}

To compute the variation of emotion in each agent at each simulation frame, first it is needed to compute the strength in which a particular emotion is transferred from agent $S$ to agent $R$. The strength of emotional contagion from agent $S$ to agent $R$ is given by $\gamma_{\mathrm{SR}}$ and calculated as the product in Equation 1:

$$
\gamma_{S R}=\varepsilon_{S} \alpha_{S R} \delta_{R} \text {. }
$$

With all $\gamma_{\mathrm{SR}}$ is possible to compute the overall strength by which emotions from all other agents in the group are received by $R$ in group $G$, indicated by $\gamma_{R}$, and defined as in Equation 2:

$$
\gamma_{R}=\sum_{S \in G|| R \mid} \gamma_{S R}
$$

As stated before, the model proposed by Bosse [2] can simulate upwards and downward emotional spirals, starting from an initial given $q_{A}$ for every agent $A$ in the scenario. Through spirals mechanisms, not only individual agents, but the whole group can get to a higher or lower level of emotion, even enabling the group to create an overall higher (or lower) emotional energy that was not present before. By carefully adjusting the parameters $\eta$ and $\beta$, the model makes it possible for a given agent $A$ to approximate any value between 0 and 1 as the interaction evolves. Each agent will reach its own emotional equilibrium within the group. Suppose A is an agent in group G, the dynamic of A's emotion level is given by Equation 3:

$$
\frac{d q_{A}}{d t}=\gamma_{A}\left[\eta_{A}\left(\beta_{A} P I+\left(1-\beta_{A}\right) N I\right)+\left(1-\eta_{A}\right) q_{A}-q_{A}\right] \text {. }
$$

Here we have the overall groups emotional influence over agent $\mathrm{A}$ denoted by $q_{A}^{*}$ and this can be computed by Equation 4:

$$
q_{A}=\sum_{S \in G|| A \mid} \omega_{S A} q_{S}
$$

A weighted sum with weights $\omega_{S A}$ computed by Equation 5:

$$
\omega_{S A}=\frac{\varepsilon_{S} \alpha_{S A}}{\sum_{C \in G \| A} \varepsilon_{C} \alpha_{C A}} .
$$

The group emotion $q_{A}{ }^{*}$ is in fact the reference for the absorption model. It represents a sort of group emotional average, and in a pure absorption situation $\left(\eta_{A}=0\right)$ the 
agents emotion will try to follow this reference. Notice that it varies in time as the emotions $\mathrm{q}_{\mathrm{s}}$ in the agents also change at every iteration. Values of $\eta_{A}$ in between 0 and 1 combine both amplification and absorption phenomena. For the pure amplification scenario $\left(\eta_{A}=1\right)$, the model brings an upward and a downward factor, biased by $\beta_{A}$ and represented respectively by $P I$, standing for Positive Influence and $N I$, standing for Negative Influence. Basically the positive influence normalizes $q_{A} *$ to fit the range $\left[q_{A}, 1\right]$, and the negative influence normalizes it to fit in the range $\left[0, q_{A}\right]$. This way, the higher the emotion level of the group, the higher will be $P I$ and the lower will be NI. At the same time, the lower $q_{A}{ }^{*}$, the lower will be $P I$ and the higher will be NI. The formulation of both influences is given by Equation 6 and Equation 7:

$$
\begin{aligned}
& P I=1-\left(1-q_{A}\right)\left(1-q_{A}\right) . \\
& N I=q_{A} q_{A} .
\end{aligned}
$$

This summarizes the formulation on Bosse's work. The results published by the authors [2] confirm the ability of the model in simulating desired emotional behaviors, such as spirals. For such reasons, it was adopted to continue in the crowd simulation scenario. The same equations used in Bosse's model for one emotion are used here. Changes to include greater number of emotions in crowd scenario are explained in Section A.

\section{A. Applying Emotion Contagion Model into Crowd Simulation Scenarios}

In the present work, experiments were made extrapolating Bosse's model [2] for a given number of emotions $N$, and scenarios containing great numbers of agents, i.e., hundreds or thousands. Enabling many emotions in the model is important because people can have many emotions, and there are many emotional models in the psychology literature [7] [8] [6], all of them suggesting more than one emotion.

Once it is considered more than one emotion in the model, the emotional contagion must be remodeled in order to deal with this variety of emotions. So, parameters related with emotions must be vectorized, each element addressing one of the scenario emotions. This way, the present model defines the emotion of an agent as a vector $q=<q_{1}, q_{2}, \ldots$, $q_{N}>$, where $q_{i}$ is the emotional level in the range $[0,1]$ of a given emotion $i$, and $N$ is the number of existing emotions in the current simulation scenario. Also, each agent expresses its own emotions to the group at a given expression level $\varepsilon$. Depending on the agents personality traits, he or she might express every emotion in different ways. A person with a happy personality might have high expressiveness of joy, but lower expressiveness of sadness or anger. This implies that $\varepsilon$ is also a vector $\varepsilon=<\varepsilon_{1}, \varepsilon_{2}, \ldots, \varepsilon_{N}>$ and the appropriate expressiveness multiplier $\varepsilon_{i}$, in the range $[0,1]$, must be applied to the respective emotion $q_{i}$. On the receiver agent one has the susceptibility of catching the emotions represented in the model by the variable $\delta$, which also lies in the range $[0,1]$. Similarly to the expressiveness $\varepsilon$, the susceptibility $\delta$ is also a vector $\delta=<\delta_{1}, \delta_{2}, \ldots, \delta_{N}>$ and each $\delta_{i}$ is the agent's susceptibility of catching others emotions $q_{i}$. Finally, the relationship between the agents must be taken under consideration. To address inserted variety of emotions, the $\alpha$ matrix must be replicated $N$ times, one for each emotion being simulated. Also, Bosse [1] [2] states that $\alpha$ must be function of attachment and distance.

Hatfield and colleagues [10] states that emotion contagion phenomena can occur when one particular emotion triggers the same emotion in other subjects. For example, if someone gets angry at some event or interest object, others, by empathy, might get anger as well at the same event. On the other hand, emotion contagion may occur under counter-contagion mechanism which means, for example, that if someone gets angry at another subject, that subject might have fear of an aggression. In this second scenario, we have a counter-contagion phenomena, where one expressed emotion drives a different emotion in the perceiver. To model this characteristic of emotion contagion, a Perception matrix with dimension $N x N$ denoted by $P$, is introduced. Its members $\rho_{i j}$ where $i$ and $j$ indicate emotion types, and the expression of one emotion might trigger perception on yet another emotion. The goal of this matrix is to perform the matrix product with the expression vector. The resulting vector, a perception vector, serves as input for agents emotional model. As a default value for this matrix an identity matrix is used, since it will not change the behavior of the single emotion model, and results in every emotion driving the same emotion, in the same level, at the agent being infected. But the values of this matrix can be adjusted to simulate other scenarios, where one expressed emotion can drive different emotions in different levels. The adjustment of the parameters on this matrix is dynamic, dependent on agent personality, situation, scenario and emotions involved in the process, which by this time, must be previously specified in order to behave and perform counter-contagion in a realistic manner.

When applying a set of $\alpha$ matrixes to the crowd simulation problem, which handles hundreds or even thousands of agents, and since the matrixes dimension is $G x G$, for huge values of $G$, one should expect that to reach prohibitive dimension. And if a number of emotions greater than one is to be considered, this prohibitive scenario can get even worse. A possible strategy is to divide the crowd in smaller groups as proposed by [12], where group behavior was introduced in the work of [4]. Also, people who knows each other and are grouped together tend to have interpersonal relationships, and tend to pay attention on each others expressions, catching each others emotions. Furthermore, contagion between groups can be done in a higher level. In other words, by dividing the crowd in groups, now contagion can be done in at least two manners: i) in an in-group manner, where agents within one group catch emotions from each other, altering the overall group emotion, and ii) in an out-group manner, where agents from one group may influence agents from another group, impacting the other group emotional tendency. The outgroup, or inter-group, possibility was not yet implemented. However, the in-group scenario was tested and results are shown in the Section V.

\section{SiMULATION RESULTS}

The scenario used to simulate the emotional contagion process is a double corridor as shown in the Figure 1. The 
red dots denote unwalkable area, i.e. walls and obstacles, the yellow dots denote walkable area. Aligned red dots denote the path agents are supposed to follow. Agents are represented by circles and the color of the circles identifies the group to which agents belong. They are labeled with identity numbers, and there is a colored ring around every agent that denotes its emotional status. Each emotion is represented by a color chosen by the user. In the scenario depicted in Figure 1, agents are programed to have three emotions each: one represented in GREEN for JOY, RED for ANGER and BLUE for SADNESS. The brightness of each color denotes the emotional level: the brighter the higher, the darker the lower. In the Figure 1, agents 3 and 4 have high levels of JOY and SADNESS, but almost zero in ANGER emotion. Agents 5 and 6, on the other hand, have high levels of ANGER and SADNESS, but JOY is in a medium state, i.e., neither too high or too low. Agent 7 is not in a group, so he will suffer no emotional contagion in the present scenario because out-group contagion is turned off. Agents cross the scenario from top to bottom and have random speed, yet within realistic range.

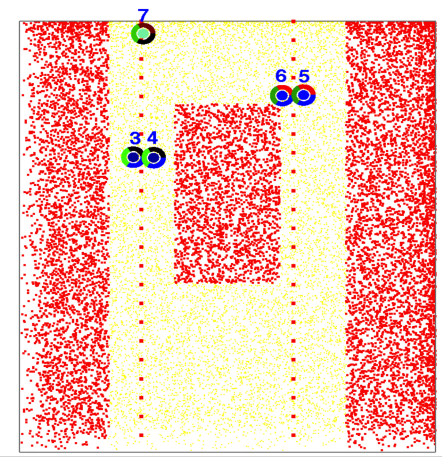

Fig. 1. Simulation scenario.

Many limitations were made in the following scenarios in order to get a first evaluation of the models responses. To begin with, the agents infect emotions only in a straight manner, i.e., no counter-contagion was performed. This allows to evaluate if contagion is properly occurring according to Bosses findings [2]. Furthermore, in the same simulated scenario all agents have the same tendency, either to absorb of amplify emotions, which does not denote any realistic scenario since people vary these tendencies. But it is simulated this way in order to depict the models behavior for each parameter setting situation. These limitations end up by creating unrealistic scenarios, but allow evaluating emotion response in particular situations in order to evaluate model's response to parameter settings.

All agents were either alone or in a two agent group, although this number can be bigger. A set of three emotions was configured for the model, represented by the colors RED denoting ANGER, GREEN denoting JOY and BLUE denoting SADNESS. When entering the scenario, grouped agents start interacting with each other, expressing their own emotions and catching each others emotions. This leads to a dynamic change in their emotional status $(q)$, influenced by others. Their initial emotional level is randomly picked, but the other parameters were adjusted to depict the following situations: i) pure absorption scenario, ii) pure amplification scenario upwards, iii) pure amplification scenario downwards, iv) absorption combined with amplification scenario upwards, and v) absorption combined with amplification scenario downwards. The parameters used for each case are detailed in the respective subsections. Graphical results were plotted using as data the instantaneous emotional level on grouped agents taken in frame-by-frame rate. The vertical axis is the emotional level, and horizontal axis is time measured in number of interactions. To ensure contagion phenomena, both expressiveness $\varepsilon$ and susceptibility $\delta$ were preset to its maximum value $\varepsilon=\delta=1$ in all simulations. Notice, again, that those are very unlikely scenarios in real life, since humans rarely tends to have same emotional expressiveness or susceptibility as the others.

\section{A. Pure Absorption Scenario}

In this simulation scenario, only the effect of absorption is observed. To do so, parameter variable $\eta$ is set to zero. This makes the result independent of the value of $\beta$, according to Equation 3. Figure $2 \mathrm{~A}$ depicts simulation results in agent $A$. Each line represents one of the three emotions (SADNESS, ANGER, andJOY) in agent $A$. Similarly, Figure 2B depicts the three emotions in agent $B$.

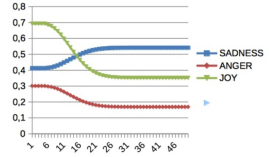

A

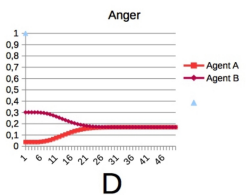

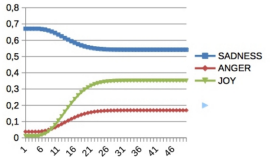

B

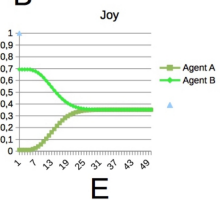

Fig. 2. Simulation results for pure absorption scenario simulating three emotions SADNESS, ANGER and JOY: A) emotional dynamic of agent $A$ during the simulation, $\mathrm{B}$ ) emotional dynamic of agent $B$ during the simulation, C) emotional dynamic of emotion SADNESS of agents $A$ and $B$, D) emotional dynamic of emotion ANGER of agents $A$ and $B$, and $E$ ) emotional dynamic of emotion $J O Y$ of agents $A$ and $B$ during the simulation. The horizontal axis denotes time elapsing in number of iterations and the vertical axis denotes emotional level. Notice how emotions tend to converge to an average point in the pure absorption model.

Figure $2 \mathrm{C}, \mathrm{D}$ and $\mathrm{E}$ shows the same results comparing each emotion separately, where one emotion is compared to the same emotion in the other agent. Each graphic is comparing the same emotion in both agents. Since no counter-contagion was programmed for this scenario, for pure absorption model is possible to see both agents $A$ and $B$ converging to a sort of average emotional level as expected.

\section{B. Pure Amplification Scenario Upwards}

In this simulation scenario, only the effect of amplification in an upwards way is observed. To do so, parameter variable $\eta$ is set to 1 , which brings $\beta$ back to the influence on the result, according to Equation 3. Yet, since this is an upwards scenario, $\beta$ is also set to 1 , which ensure maximum PI. Figure 3A depicts simulation results in agent $A$. Each line represents one of the three emotions (SADNESS, ANGER, and JOY) in agent $A$. Similarly, Figure 
3B depicts the three emotions in agent $B$. As expected from the adjusted parameter, an upward spiral is observed in every curve. Emotional energy is created from agents interaction and the emotions tend to reach their maximum.
A

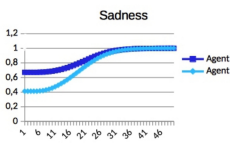

C

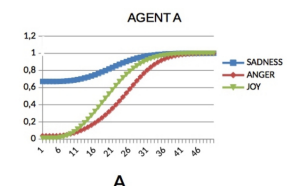

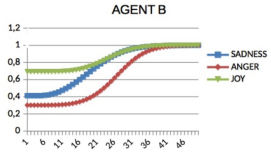

B

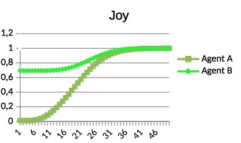

$\mathrm{E}$
Fig. 3. Simulation results for pure amplification scenario with upwards tendency simulating three emotions SADNESS, ANGER and JOY: A) emotional dynamic of agent $A$, B) emotional dynamic of agent $B, \mathrm{C}$ ) emotional dynamic of SADNESS on agents $A$ and $B$, D) emotional dynamic of ANGER on agents $A$ and $B$, and E) emotional dynamic of JOY on agents $A$ and $B$. The horizontal axis denotes time elapsing in number of iterations and the vertical axis denotes emotional level. Notice how emotions tend to grow in time reaching its maximum.

Figure 3C shows SADNESS evolution in both agents $A$ and $B$ as the simulation advances. Similarly, Figure 3D shows their ANGER levels, and Figure 3E shows their JOY levels. As expected, amplification model with tendency to rise emotions results in upwards spirals. Even emotions starting in lower level, such as observed in emotion $A N G E R$, tend to grow up to their maximum as the simulation evolves.

\section{Pure Amplification Scenario Downwards}

In this simulation scenario, only the effect of amplification in a downwards way is observed. To do so, parameter variable $\eta$ is set to 1 for amplification bringing $\beta$ back to the influence on the result, according to Equation 3. In a downwards scenario, $\beta$ must set to zero, which ensure maximum NI. Figure 4A depicts simulation results in agent $A$. Each line represents one of the three emotions (SADNESS, ANGER, and JOY) in agent $A$. Similarly, Figure 4B depicts the same three emotions in agent $B$. This time emotional levels go down to zero as the simulation evolves.

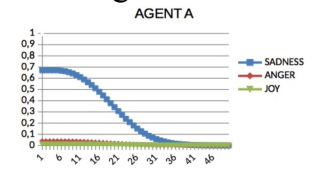

A

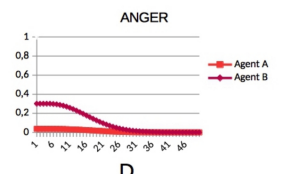

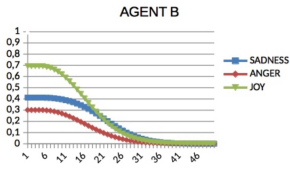

B

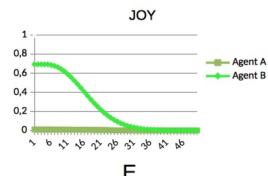

$\mathrm{E}$ c

Fig. 4. Simulation results for pure amplification scenario set to

downwards tendency simulating three emotions BLUE, RED and GREEN:

A) emotional dynamic of agent $A$, B) emotional dynamic of agent $B, \mathrm{C}$ )

emotional evolution of SADNESS on agents $A$ and $B, D$ ) emotional

dynamic of $A N G E R$ on agents $A$ and $B$, and E) emotional dynamic of

emotion JOY on agents $A$ and $B$. The horizontal axis denotes time elapsing

in number of iterations and the vertical axis denotes emotional level in all

graphics. Notice how emotions tend to decay in time reaching its minimum no matter how high they have been initialized.

Figure 4C, D and E shows the same results comparing each emotion separately, where one emotion is compared to the same emotion in the other agent. Each graphic is comparing the same emotion in both agents. As expected, amplification model with tendency to lower emotions results in downwards spirals. Even emotions starting in higher levels, such as observed in emotion SADNESS tend to decay down to their minimum.

\section{Absorption mixed with Amplification Scenario Upwards}

In this simulation scenario, the effect of amplification in an upwards way mixed with absorption is observed. To do so, parameter variable $\eta$ is set to $1 / 2$, enabling same power to both amplification and absorption portions of Equation 3 . For the amplification portion, $\beta$ must be set to 1 , since this is an upwards scenario. As in the other cases, both expressiveness $\varepsilon$ and susceptibility $\delta$ were preset to its maximum value $\varepsilon=\delta=1$. Figure $5 \mathrm{~A}$ depicts simulation results in agent $A$, and Figure 5B depicts the three emotions in agent $B$.

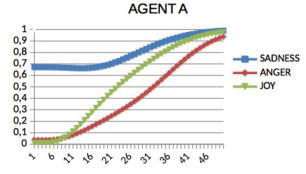

A

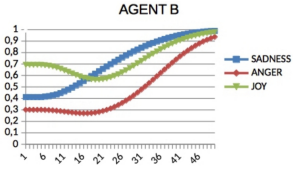

$\mathrm{B}$

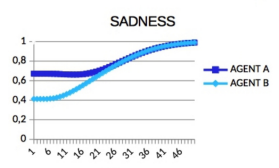

C

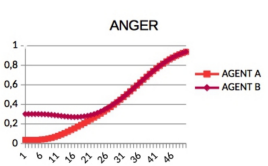

D

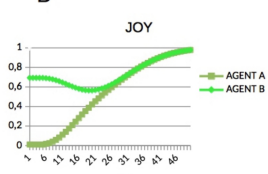

Fig. 5. Simulation results for amplification combined with absorption scenario set to upwards tendency, simulating three emotions SADNESS, ANGER and JOY: A) emotional dynamic of agent $A, \mathrm{~B}$ ) emotional dynamic of agent $B, C$ ) emotional dynamic of SADNESS on agents $A$ and $B$, D) emotional dynamic of $A N G E R$ on agents $A$ and $B$, and E) emotional dynamic of $J O Y$ on agents $A$ and $B$. The horizontal axis denotes time elapsing in number of iterations and the vertical axis denotes emotional level. In this scenario, first emotions tend to meet each other in a sort of average, and then upwards spirals are observed.

Figure 5C, D and E shows the results of each emotion separately. Giving special attention to agents $B$ levels of $J O Y$ in Figure 5E, it can be noticed that in the beginning of the simulation this value starts dropping. This happens because emotional levels tend to meet each other driven by the absorption portion of the model. As soon as those emotional levels get close enough, upward spirals drive $J O Y$ on agent $B$ to its maximum, along with $J O Y$ on agent $A$.

\section{E. Absorption mixed with Amplification Scenario Downwards}

In this simulation scenario, the effect of amplification in a downwards way along with absorption is observed. As in the last scenario, parameter $\eta$ is set to $1 / 2$. For the amplification portion, $\beta$ must be set to zero, since this is a downwards scenario. As in the other cases, both expressiveness $\varepsilon$ and susceptibility $\delta$ were preset to its maximum value $\varepsilon=\delta=1$. Figure 6A depicts simulation results in agent $A$, and Figure 6B depicts the three emotions in agent $B$. 


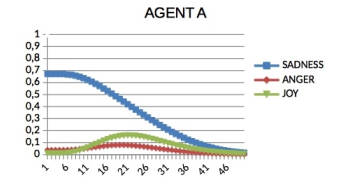

A

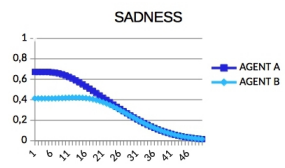

C

D

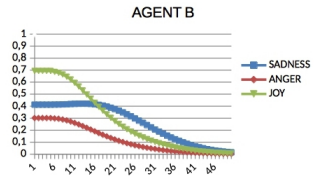

B

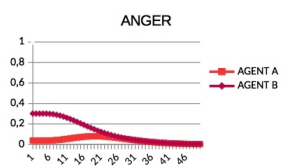

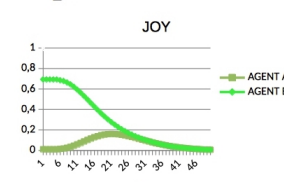

$\mathrm{E}$
Fig. 6. Simulation results for amplification combined with absorption scenario set to downwards tendency, simulating three emotions SADNESS, ANGER and JOY: A) emotional dynamic of agent $A, B$ ) emotional dynamic of agent $B, C$ ) emotional dynamic of SADNESS on agents $A$ and $B$, D) emotional dynamic of $A N G E R$ on agents $A$ and $B$, and $E$ ) emotional dynamic of $J O Y$ on agents $A$ and $B$. The horizontal axis denotes time elapsing in number of iterations and the vertical axis denotes emotional level. In this scenario, first emotions tend to meet each other in a sort of average, and then downwards spirals are observed.

Figure 6C, D and E shows the results of each emotion separately. Now looking at agents $A$ levels of $J O Y$, it can be noticed that in the beginning of the simulation this value starts rising, trying to meet $B$ level of JOY driven by absorption. Nevertheless, since agents parameter $\beta$ is zero, emotional levels inevitably decay.

\section{F. Result Analysis}

In this section, some simulation scenarios were designed to verify emotion contagion in crowd agents as they navigate in a virtual world. Model parameters were carefully adjusted to observe some desired behaviors. Parameter $\eta$ was changed in order to account with both pure absorption, pure amplification and a mix of both situations. At the same time, parameter $\beta$ was either fixed on 1, for upward spirals, or zero for downwards spirals. As expected, emotion convergence and emotional spirals were observed.

Nevertheless, it is important to notice that those scenarios are not very realistic. Usually, people have different tendencies for each emotional type. Active expressive people might have upward spirals for emotions like $J O Y$ and downward spiral to attenuate emotions like $S A D N E S S$. This scenario is much more realistic than the scenarios simulated here, where all emotions in all agents have the same tendency. More diversification in the population leads to less predictability, but yet more realistic results. Scenarios showing divergence in emotional stabilization can also be observed within the model, specially when the model is operating in amplification mode and some of the agents tend to upward spirals, while others tend to downward spirals. In those cases, agents emotion get stable in very different emotional levels from one agent to the other. To analyze scenarios like that, it is important to recreate basic characteristics of personalities, in order to determine a particular emotion tendency (upward or downward) and its consistency with agents personality. Also, mixing agents with such different emotional tendencies may result in more diverse responses, where agents can even stabilize their emotional level in different final values. Furthermore, scenarios with countercontagion must be considered. Since no evaluation was yet made in order to classify emotional tendencies and relate them with personalities, no such results were analyzed at the present moment.

\section{CONCLUSIONS AND FUTURE WORK}

Analyzing results found so far, the emotion computational model developed by Bosse [2] prove itself to have enough flexibility to simulate many contagion situation, considering both personal aspects (such as susceptibility), relational aspects (denoted by $\alpha$ ) and external aspects (such as expressiveness). The extrapolation of the model to numerous emotions also proved promising, allowing every emotion to behave independently, with its own parameters. Finally, the model requires simple computational resources, which can be an issue in crowd simulation scenarios, although further stressing tests, essentially increasing number of agents in groups are still necessary to retrieve computational limitations of the model, if any.

For future works, a main concern is filling the perception matrix $P$ with realistic values. Counter-contagion may happen in many ways, and is deeply dependent on the scenario. On behalf of that, a plausible hypothesis is that the values of matrix $P$ are dynamic, and change from time to time. Another concern is that the nature of emotions matter at this time, in other words, counter contagion mechanisms for joy driving other emotions may work in a different manner than counter contagion for anger driving other emotions. The situations where counter contagion occurs must be addressed as well as the types of emotion such situation may induce into a person. Furthermore, it must be mapped the strength that induced emotion is perceived in a counter-contagion scenario. Other hypothesis might even consider one particular emotion driving more than one emotion in the target subject.

Some works like [14] have mapped emotional traits and linked them to simulation parameters. Efforts must be made to translate emotional traits to emotional tendency parameters like expressiveness, susceptibility, tendency for upward or downward spirals or even tendency to diverge in emotional level. By doing that, emotional behavior can be consistent with agent personality.

To create emotional perception in the crowd, agents emotional status must have impact on its behavior. Since crowd simulation models usually simulate agents seeking goals and navigating, emotional status should impact those mechanisms. If there is any correlation between agent speed and its emotions, that should be mapped. Furthermore, agent might want to change its goal according to a change in emotional state as in situations of panic. Specially if the panic causing event is between the agent and its original goal, there might be a plausible change in agents desires and goals.

Some works like \cite\{Ramos:2014\} explored body expression of agents in crowds to denote their emotional state. Studies were made to measure the perception of an external observer on emotion expression and emotion contagion in this crowd. Although their emotional model 
and emotion contagion model are extremely simple, the work focuses on the animation of characters and body expression itself. Integrating such attempts in a scenario were a more dynamic emotional model rules, possibly leads to different perception in agents emotions. Having emotional information in crowd agents can drive proper animation on agents avatar, and an external observer (a human user) may have perception of emotion and emotion contagion within the crowd.

\section{- ACKNOWLEDGMENTS}

Authors thank LTSI-CNRS and PUCRS for supporting the research. They also thank CAPES (grant 7156-14-6 PDSE) for the financial support to this research.

\section{- REFERENCES}

[1] Bosse, T., Duell, R., Memon, Z., Treur, J., and van der Wal, C. (2009). A multi-agent model for emotion contagion spirals integrated within a supporting ambient agent model. In Yang, J.J., Yokoo, M., Ito, T., Jin, Z., and Scerri, P., editors, Principles of Practice in Multi-Agent Systems, volume 5925 of Lecture Notes in Computer Science, pages 48-67. Springer Berlin Heidelberg.

[2] Bosse, T., Duell, R., Memon, Z., Treur, J., and van der Wal, C. (2015). Agent-based modeling of emotion contagion in groups. Cognitive Computation, 7(1):111-136.

[3] Carretero, M. R., Peters, C., and Qureshi, A. (2014). Modelling emotional behaviour in virtual crowds through expressive body movements and emotion contagion. In Proceedings of SIGRAD 2014, Visual Computing, June 12-13, 2014, G“oteborg, Sweden, pages 95-98.

[4] de Lima Bicho, A. (2009). Da modelagem de plantas `a din^amica de multidºes: um modelo de animac, ao comportamental bio-inspirado. PhD thesis, Universidade Estadual de Campinas - UNICAMP, Campinas.

[5] Durupinar, F., Allbeck, J., Pelechano, N., and Badler, N. (2008). Creating crowd variation with the ocean personality model. In Padgham, Parkes, M. and Parsons, editors, Proceedings of the 7th International Joint Conference on Autonomous Agents and Multiagent Systems(AAMAS 08), pages 1217-1220, Estoril, Portugal. International Foundation for Autonomous Agents and Multiagent Systems.

[6] Ekman, P. (1992). An argument for basic emotions. Cognition and Emotion, 6(3):169-200.
Eysenck, H. J. and Eysenck, M. W. (1985). Personality and Individual Differences: A Natural Science Approach. Plenum Press, New York, NY.

[8] Goldberg, L. R. (1990). An alternative "description of personality": The big-five factor structure. Journal of Personality and Social Psychology, 59(6):1216-1229.

[9] Guy, S. J., Kim, S., Lin, M. C., and Manocha, D. (2011). Simulating heterogeneous crowd behavior using personality trait theory. In Bargteil, A. and van de Panne, M., editors, Eurographics / ACM SIGGRAPH Symposium on Computer Animation, SCA '11, pages 43-52, New York. ACM.

[10] Hatfield, E., Cacioppo, J. T., and Rapson, R. L. (1994). Emotional Contagion. Cambridge University Press, New York.

[11] Helbing, D. and Molnar, P. (1995). Social force model for pedestrian dynamics. Physical Review E, 51:4282.

[12] Hocevar, R. (2013). Simulation of inter and intra group behaviors usging semantic virtual environments. Master's thesis, Pontif'ıcia Universidade Cat'olica do Rio Grande do Sul PUCRS.

[13] Hurst, M. (2014). Collective behavior: Crowd types, mobs \& riots. (accessed October 10th, 2014).

[14] Kappadia, M., Pelechano, N., Guy, S., Allbeck, J., and Chrysanthou, Y. (2014). Simulating heterogeneous crowds with interactive behaviors. Eurographics 2014.

[15] Pelechano, N., Allbeck, J. M., and Badler, N. I. (2007). Controlling individual agents in high-density crowd simulation. In Proceedings of the 2007 ACM SIGGRAPH/ Eurographics Symposium on Computer Animation, SCA '07, pages 99-108, Aire-la-Ville, Switzerland, Switzerland. Eurographics Association.

[16] Reynolds, C. W. (1987). Flocks, herds and schools: A distributed behavioral model. In SIGGRAPH '87: Proceedings of the 14th annual conference on Computer graphics and interactive techniques, pages 25-34, New York, NY, USA. ACM.

[17] Runions, A., Fuhrer, M., Lane, B., Federl, P., Rolland-Lagan, A.G., and Prusinkiewicz, P. (2005). Modeling and visualization of leaf venation patterns. In SIGGRAPH '05: ACM SIGGRAPH 2005 Papers, pages 702-711, New York, NY, USA. ACM.

[18] Runions, A., Lane, B., and Prusinkiewicz, P. (2007). Modeling trees with a space colonization algorithm. In Proceedings of the Eurographics Workshop on Natural Phenomena, pages 63-70, Aire-la-Ville, Switzerland. Eurographics Association.

[19] Thalmann, D. and Musse, S. R. (2013). Crowd Simulation. Springer-Verlag London Ltd, London, UK. 